\title{
LA DOCTRINA DE LAS RAZONES SEMINALES ASUMIDA POR DUNS ESCOTO
}

\author{
EnRiQue Santiago Mayocchi \\ Universidad Católica Argentina, Buenos Aires
}

\section{Resumen}

La doctrina de las rationes seminales propuesta por el Doctor de Hipona fue para muchos pensadores un refugio que permite explicar la generación en el mundo físico. Duns Escoto retoma el pensamiento agustiniano, y analiza las posturas que le precedieron. El Doctor Sutil desarrolla una respuesta propia, que lo lleva a completar su doctrina de los elementos y explicar la generación natural derivando el tema, en perspectiva antropológica, hacia la creación del alma racional en un cuerpo. En el presente trabajo analizaremos los diferentes pasajes de la obra escotista donde se profundiza este tema, y también incluiremos algunas referencias a su teoría de la causalidad en el mundo natural.

Palabras clave: razones seminales, Duns Escoto, creación, generación.

\begin{abstract}
The doctrine of the rationes seminales suggested by the Doctor of Hippo was a shelter for many thinkers, who allows to explain the generation in the physical world. Duns Scotus returns to the Augustinian thought and analyzes the postures of those who had preceded him. Develops an answer of his own which leads him to complete his doctrine of the elements and to explain the natural generation diverting the subject into an anthropological perspective towards the creation of the rational soul in a body. In this paper we analyze the different passages of Scotus's work where this topic is deepened, and also include some references to his theory of causality in the natural world.
\end{abstract}

Keywords: seminal reasons, Duns Scotus, creation, generation.

El tema de la generación de nuevos seres en el mundo natural ha sido una preocupación constante a lo largo de la historia de la filosofía, incluso

Recibido: 22/12/2014. Aceptado: 09/06/2015. 
derivando hacia explicaciones específicas en distintos ámbitos de la ciencia, como la química o la biología. Comprender la continuidad de las especies a través de diversas generaciones y la aparición de entidades nuevas, motivaron la búsqueda de una respuesta racional al hecho empírico. En el mundo antiguo podemos encontrar en Anaxágoras y Demócrito el primer paso hacia lo que luego se denominaría como teoría de las rationes seminales, esbozada por los Estoicos ${ }^{1}$.

En los albores del Medioevo, Agustín de Hipona retoma desde el pensamiento creacionista dicha teoría. En varias de sus obras, pero sobre todo en los comentarios al Génesis, desarrolla lo que para él es la solución tanto a la generación natural como para la actividad divina del Creador. En el presente artículo, nos centraremos en la recepción que hace Duns Escoto de esta cuestión, no tratada exhaustivamente en el marco de su filosofía de la naturaleza, y que es de suma importancia para comprender los temas con los que se relaciona como su concepción sobre la teoría hilemórfica, la composición de los mixtos desde los elementos, la causalidad natural y su conexión con la acción creadora.

El desarrollo en particular sobre las rationes seminales lo tomaremos de las Quaestiones super Metaphysicorum (VII, q. 12), Lectura in Sententiarum (II d. 18), y las Reportata parisiensia (II d. 18). Seguiremos el esquema que suele proponer el mismo Doctor Sutil en sus tratados, por ello primero pasaremos revista a las doctrinas de otros pensadores, algunas de las cuales proponen las rationes seminales para evitar recurrir a la creación en la explicación del cambio substancial en el mundo físico (1.a); y otras que defienden dichas rationes como principios activos para fundamentar el obrar natural en el mundo físico (1.b). En un segundo momento desarrollaremos la opinión propia de Duns Escoto, trazando un paralelismo con el Doctor de Hipona, sobre los conceptos utilizados (2.a); para atender luego el análisis propiamente escotista sobre la generación en los vivientes (2.b) y considerar por último si hay rationes seminales en los seres inertes (2.c).

\section{Posiciones revisadas por el Doctor Sutil}

\section{a. Para salvar la generación natural de la creación de formas}

Desde la teoría de la composición hilemórfica, formulada por Aristóteles, la producción de nuevos seres en el mundo físico se presenta como

${ }^{1}$ Cfr. M. I. Colish, The Stoic tradition from Antiquity to the Early Middle Ages, Leiden, Brill, 1985, vol. 1, pp. 31-35. 
la aparición de nuevas formas que previamente no existían. La materia, actualizada por una forma específica, hace capaz al sujeto de transformarse en una substancia de naturaleza distinta a la que es. Ahora bien, si toda generación es por definición la aparición de nuevas formas substanciales, éstas deben empezar a existir una vez corrompida su forma precedente, pero ¿hay conexión entre ellas? Desde la experiencia nos es evidente que sí, y sobre todo en el caso de los vivientes, cuando reproducen un individuo de su misma especie pero distinto en número respecto de sus progenitores. Si la respuesta es negativa, y no hay relación entre ellas, se concluirá que la nueva forma es creada, por ello muchos pensadores medievales han relacionado la doctrina de las rationes seminales con formalidades preexistentes en la materia, que actúan junto con otro agente para generar la nueva forma. En las Quaestiones super Metaphysicorum, Escoto introduce el estudio de las diferentes versiones sobre el presente tema diciendo que "es una opinión famosa el que haya en la materia algo esencialmente distinta de ella (...), de lo cual se hace una [nueva] forma y se la llama incoación, o parte de la forma (...)" 2 . Y a continuación presenta cuatro modelos diferentes en los que se sostiene esa opinión sobre las rationes seminales como formas incoadas en la materia: ya sea que se entienda una forma general (Egidio Romano) que actúa como ratio seminalis respecto del género próximo en la nueva substancia; o como propiamente formas incoadas en la materia, ya sea en parte (Roger Marston, Mateo de Acquasparta), o toda la forma -unida o no a la materia- (Buenaventura); o toda la forma preexistiendo como una especie de cualidad (Jacobo de Viterbo).

¿Es correcto establecer formas preexistentes para no introducir la creación en la generación natural? Este es el primer problema que revisa Duns Escoto en su tratamiento de las posturas de la época sobre las rationes seminales, a partir de la explicación que ofrece Enrique de Gante sobre el tema ${ }^{3}$, y lo formula preguntándose "si la razón seminal en la materia natural es parte de la forma a educir" 4 . El Doctor Solemne entiende que para no derivar la explicación de la generación natural como una continua creación de formas, hay que establecer en la materia las posibles formas futuras,

2 I. Duns Scotus, Quaestiones Super Libros Metaphysicorum Aristotelis, New York, Franciscan Institute Publications, 1997, VII q. 12 n. 12. Los editores apuntan como representantes de esta opinión famosa a Roberto de Groseteste, Buenaventura y Alberto Magno.

${ }^{3}$ Cfr. Henricus de Gandavo, Quodlibet IV, Lovaina, Leuven University Press, 2011, q. 14, "Utrum in materia sit ratio seminalis, quae est formae inchoatio".

${ }^{4}$ I. Duns Scotus, Lectura in II Sententiarum, Vaticano, Typis Polyglottis Vaticanis, 1993, vol. XIX, d. 18 q. 1-2 n. 1. 
existiendo de manera latente y esperando por su actualización. Por esas formas debemos entender las rationes seminales que hay en la materia, como potencias activas que educen una nueva forma en acto, y por consiguiente, la generación de una nueva substancia. Si, por el contrario, la materia no interviene en la producción de una nueva forma, entonces ésta será creada ${ }^{5}$.

Esto se explicaría admitiendo que las formas substanciales tienen grados, y así en cuanto ratio seminalis una forma se encontraría existiendo toda ella en un grado menor al que tendrá luego de ser completamente actualizada por el movimiento de generación. O de otra manera, como si la razón seminal fuese parte de la forma que se generará, pero que existe previamente - de manera latente - en el ser que puede obrar para generarla. Sin embargo, para el Doctor Sutil, ninguna de la dos posturas son adecuadas por lo que se mostrará contrario a aceptar, al menos como principio general, tales rationes seminales en los seres naturales.

Respecto de la primera posibilidad, si la forma substancial admite grados tendría que estar toda ella en el agente que actúa, pero en grado menor al que tendrá luego. Sin embargo, "el segundo grado de la forma, que es más perfecto, pertenece a la virtud de la acción del agente, de otra manera el que genera nada nuevo causaría" " y se terminaría disolviendo la generación en una mera alteración. Por ello, no conviene poner rationes seminales como formas imperfectas que puedan actualizarse. Además, en diversos momentos de su obra, Escoto niega que se puedan dar grados en las formas substanciales ${ }^{7}$, ya que las considera en sí mismas individuales, aún cuando se encuentran componiendo una substancia junto con la materia.

Respecto de la segunda doctrina, es decir, que parte de la forma a educir preexista, tampoco resulta conveniente. Ya que, si en parte hay algo preexistente, esto significa que también una parte nueva se produce enteramente, y por lo tanto no se evita la creación, porque esta parte que se produce no será causada por el agente natural que posee la parte preexistente de la forma a generar, sino que deberá ser causada ex nibilo, luego será creada. Y si no se hace algo enteramente nuevo, caemos nuevamente, afirma el Doctor Sutil, en la negación de la generación y la afirmación de una simple alteración ${ }^{8}$. También se evidencia lo inconveniente de esta postura

${ }^{5}$ Cfr. R. Cross, The physics of Duns Scotus, Oxford, Clarendon Press, 1998, pp. 42-43 http://dx.doi.org/10.1093/acprof:oso/9780198269748.001.0001.

${ }^{6}$ I. Duns Scotus, Lectura II, op. cit., d. 18 q. 1-2 n. 60.

${ }^{7}$ Cfr. I. Duns Scotus, Ordinatio, Vaticano, Typis Polyglottis Vaticanis, 2008 vol. XI, IV d. 4 q. 11 n. 44; QQ. Metaph., op. cit., VIII q. 2-3.

${ }^{8}$ Cfr. I. Duns Scotus, QQ. Metaph., op. cit., VII q. 12 n. 28. 
analizando la ratio de la forma, es decir, las características propias que tiene cuando preexiste en la materia. En la generación se da el pasaje hacia una nueva forma, pero puede suceder que aquella posea una ratio distinta a la del generante, por ejemplo desde el fuego hacia el agua. En este caso la generación se da entre formas contrarias, pero ¿cómo puede encontrarse parte de la forma del agua preexistiendo en la forma del fuego si son contrarias? Es contradictorio que el fuego sea agua, aunque sólo en parte, ya que sus cualidades son esencialmente distintas. Luego, no es conveniente sostener la existencias de formas incoadas, ya sea en parte o toda, porque no logran explicar de manera suficiente lo que se propone la teoría.

Por último, Duns Escoto resuelve una objeción que se le podría hacer "¿de qué manera se salva que la forma no sea creada, si nada de ella preexiste?" . Analizaremos más adelante cómo se entiende el concepto creación en la filosofía escotista, pero basta ahora con decir que es la causalidad primera, y que su género no presupone ninguna otra causa ni materia. En cambio, la causalidad del mundo natural sí presupone otra causa, ya sea eficiente (concurriendo con la acción divina) o material (considerándola como una circunstancia causal). En la filosofía natural del Doctor Sutil, la materia puede ser considerada como potencia objetiva, esto es, no existiendo en un sujeto, pero pudiendo existir en él (como en el caso del creabile); pero también en potencia subjetiva, puesto que ella posee una cierta actualidad -la más ínfima que puede haber- que la hace capaz de recibir una forma enteramente nueva y formar así un compuesto substancial ${ }^{10}$. Este segundo modo de entender la materia es el que se da en el mundo físico y el que permite al Escocés explicar la generación y corrupción sin caer en creación y aniquilación. Además, esto es posible porque en el pensamiento escotista, se admite la pluralidad de formas ordenadas de modo jerárquico.

\section{b. Para salvar la generación de un acto violento}

Otra pregunta que introduce el Doctor Sutil es "si la razón seminal en la materia natural es una potencia activa" ${ }^{11}$. La cuestión apunta a identificar la generación con un movimiento propio de la naturaleza (como principio intrínseco de movimiento, según la definición aristotélica) de los seres naturales. Si en la materia no se encuentra esta potencia activa, que serían las rationes seminales, se podría concluir que la producción de un nuevo

\footnotetext{
${ }^{9}$ I. Duns Scotus, Lectura II, op. cit., d. 18 q. 1-2 n. 70.

${ }^{10}$ Cfr. I. Duns Scotus, Lectura II, op. cit., d. 12 q. única n. 30; n. 37; nn. 48-51.

${ }^{11}$ I. Duns Scotus, Lectura II, op. cit., d. 18 q. 1-2 n. 5.
} 
ser está dada por una causa externa y así afirmar que es un movimiento violento respecto a la naturaleza. Escoto asume la doctrina del movimiento natural y violento del Estagirita:

Si hay un movimiento violento, entonces tiene que haber también un movimiento natural (porque el movimiento violento es contrario a la naturaleza y el movimiento contrario a la naturaleza es posterior al que es según la naturaleza, de manera que, si no hubiese un movimiento según la naturaleza en cada uno de los cuerpos naturales, no habría tampoco ninguno de los otros movimientos) $)^{12}$.

En la física aristotélica, el movimiento natural se descubre por la tendencia que tienen los seres a conseguir ciertos fines determinados por su estructuración dinámica específica. De allí que el término naturaleza se entienda como el principio de actividad que se encuentra en el interior de los entes, distinto para cada uno de ellos según la configuración que posean. El ejemplo que utiliza Aristóteles para ilustrar esto son los cuerpos pesados y ligeros ${ }^{13}$, que por su constitución apetecen de modo natural dirigirse hacia abajo o arriba, respectivamente. Por otro lado, el movimiento natural tiene prioridad sobre el movimiento violento, ya que éste último supone el primero, según se desprende del texto antes citado. El movimiento violento se distingue por ser causado desde el exterior, de tal manera que se impide al que lo padece el desarrollo de su propia natura y los fines establecidos por ella. "El movimiento violento es, pues, un movimiento per se, que nunca le corresponde a un ser por sí mismo, sino siempre per accidens; es un movimiento propio de un ser otro que aquel al que corresponde; es, en definitiva, el movimiento propio de lo impropio" ${ }^{14}$.

Para argumentar que la generación en los seres físicos no es algo violento o forzado sino adecuado a su naturaleza, es decir, un movimiento que tiene su origen en ellos mismos, Duns Escoto denuncia que se ha intentado introducir en la materia un principio activo que asegure el proceso de producción de nuevas formas sin necesidad alguna. Esta solución no es satisfactoria, según su opinión, ya que se debe distinguir en los seres un principio activo y otro pasivo cuando realizan movimientos naturales ${ }^{15}$. El primero corresponde a la inclinación que posee un ente respecto de ciertos fines dados por su naturaleza, en este caso ciertos quiere decir que son muchos y

${ }^{12}$ Aristóteles, Física, Madrid, Gredos, 1999, IV 8 215a 1-5.

${ }^{13}$ Cfr. ibidem, VIII 4 255b 13-25.

${ }^{14}$ A. Quevedo, "El movimiento accidental en Aristóteles", Anuario Filosófico 22/2 (1989) 166.

${ }^{15}$ Cfr. I. Duns Scotus, Lectura in II Sententiarum, Vaticano, Typis Polyglottis Vaticanis, 1982, vol. XVIII, d. 2 p. 2 q. 5-6 n. 323. 
variados. Luego, el principio activo indicará el origen del movimiento hacia los fines propios, lo que determina la eficiencia del movimiento. Pero cuando se intenta definir un movimiento natural, no hace falta éste último sino sólo el primero, es decir, el principio pasivo ${ }^{16}$, ya que así es como interpreta el Escocés la definición aristotélica de naturaleza. En efecto, dice Aristóteles que "la naturaleza en un principio y causa del movimiento o del reposo en la cosa a la que pertenece primariamente y por sí misma, no por accidente" ${ }^{17}$. El principio que posee un ser de manera primaria es pasivo, en cuanto inclinación, y se especifica en el actuar concreto, por su principio activo. Este último, aunque se posea, no es primero ya que son separables el motor de la acción y lo que es movido, e incluso en el caso del médico que se cura a sí mismo, la concurrencia de ambos es per accidens ${ }^{18}$. Por tanto, si no es necesario establecer el principio activo como constitutivo necesario del movimiento natural, no se tienen que establecer las rationes seminales como potencias activas en la materia para evitar explicar la generación como un movimiento violento, ya que no se debe multiplicar el ser sin necesidad ${ }^{19}$.

Por último, el Doctor Sutil da respuesta a ciertas objeciones tomadas de textos aristotélicos sobre el movimiento natural y su principio activo, comparándolos con el arte, lo involuntario y lo fortuito. Cuando Aristóteles diferencia el modo de hacer propio del arte y de la naturaleza, afirma que "las cosas por naturaleza son aquellas que, movidas por un principio interno llegan a un fin" ${ }^{20}$, y este principio puede ser tomado como activo, es decir, eficiente y no meramente pasivo. Según interpreta Escoto, decir que las cosas naturales tienen un principio activo, que es la razón de su eficiencia no es faltar a la verdad. Sin embargo, ese principio no denota la necesidad natural del movimiento, sino sólo la realización actual, que es posible gracias a la inclinación natural pasiva que posee primariamente ese ente. El otro ejemplo del Estagirita son los actos involuntarios, que deben entenderse como "aquello cuyo principio es externo, sin que el hombre intervenga forzadamente" 21 . Parecería que, si no hay potencia activa en la materia, el principio de movimiento sería externo y por tanto violento. Sin embargo, el Maestro escocés aclara que en los seres naturales no podemos aplicar tal afirmación porque la inclinación pasiva explica la posibilidad de

${ }^{16}$ Cfr. I. Duns Scotus, Lectura II, op. cit., d. 18 q. 1-2 n. 74.

${ }^{17}$ Aristóteles, Física, op. cit., II 1 192b 20-23.

${ }^{18}$ Cfr. Aristóteles, Física, op. cit., II 1 192b 24-26; I. Duns Scotus, Lectura II, op. cit., d. 18 q. $1-2$ n. 75.

${ }^{19}$ Cfr. I. Duns Scotus, Lectura II, op, cit., d. 18 q. 1-2 n. 64.

${ }^{20}$ Aristóteles, Física, op. cit., II 8 199b 15-17.

${ }^{21}$ Aristóteles, Ética a Nicómaco, Madrid, Gredos, 1985, III 1 1110b 15-17. 
buscar nuevas formas por medio de la generación, tal posibilidad no requiere la necesaria realización de la misma. Por último, parece que la naturaleza obra de manera eficiente porque "la casualidad y la suerte son causas de cosas que, pudiendo ser causadas por la inteligencia o por la naturaleza, han sido causadas accidentalmente por algo"22. Una vez más, el Doctor Sutil aclara que allí debe entenderse el concepto naturaleza como aquello determinado a un fin, y no a muchos, como en el caso del intelecto. Pero esta interpretación no debe confundirse con la otra posible, que contrapone lo natural a lo violento. En definitiva, no se deben establecer en la materia natural ninguna potencia activa que cumpla funciones a modo de rationes seminales. Supuesto este primer aspecto, desarrollaremos la propuesta escotista sobre la cuestión.

\section{Doctrina escotista sobre las rationes seminales}

\section{a. La conceptualización de Agustín de Hipona asumida por Duns Escoto}

El primer paso que ofrece Escoto, antes de dar su propia solución a la cuestión, es el análisis de los vocablos semen y ratio seminalis, según el pensamiento de Agustín, haciendo referencias explícitas a pasajes de las obras Sobre la Trinidad y Comentario literal al Génesis. Como ya hemos dicho antes, estas nociones tienen su origen en los Estoicos, incluso el Doctor de Hipona pudo haberlas reformulado desde Anaxágoras o Plotino ${ }^{23}$.

Es manifiesta su intención de explicar la creación como aquel acto por el que todo fue creado de manera simultánea por la voluntad divina, quien dio existencia ex nibilo a los seres del mundo material. En su Comentario literal al Génesis, se pregunta si la creación se produjo en un mismo instante o a intervalos de tiempo ${ }^{24}$, y la respuesta viene dada en relación al pasaje bíblico cuando se afirma "el que vive eternamente todo lo creó al mismo tiempo" 25 . Por tal motivo, según Agustín, debe entenderse el relato de los seis días como un solo instante, en el cuál todo fue producido por el Creador, de una sola vez y para siempre. Luego, queda dar una explicación adecuada al

${ }^{22}$ Aristóteles, Física, op. cit., II 6 198a 5-6.

${ }^{23}$ Cfr. A. C. Crombie, Historia de la Ciencia: De San Agustín a Galileo, Madrid, Alianza, 1996, vol. 1, p. 41.

${ }^{24}$ Cfr. Agustín de Hipona, Comentario literal al Génesis, Pamplona, Eunsa, 2006, IV 33.51. "¿Acaso también lo que se creaba (...) fue hecho todo al mismo tiempo? ¿O mejor por intervalos de tiempo cada uno en el día fijado?”.

${ }^{25}$ Biblia de Jerusalén, Bilbao, Descleé de Brouwer, 1999, Eclesiástico 18, 1. 
surgimiento de nuevos seres corpóreos a lo largo del tiempo, los cuales no se encontraban antes pero que ahora existen. ¿Cómo hacer compatible la creación simultánea con la novedad en el mundo material? Aquí es donde se hace presente la doctrina de las rationes seminales, ya que los seres creados no pueden crear (esto le compete solamente a la omnipotencia divina), ni tampoco el Creador obra de manera sucesiva en el tiempo:

Tan fácil creó Dios todo como lo dotó de movimiento en la medida más eficaz, para que esto que ahora vemos moverse en intervalos de tiempo para cumplir las acciones propias de cada naturaleza, tenga origen en las razones que Dios ha dispuesto de manera seminal en el momento de crearlas ${ }^{26}$.

La original solución del Hiponense sobre la aparente contradicción entre creación simultánea y movimiento del mundo físico que conlleva novedades, consiste en afirmar que hay en la materia virtualidades que guían el desarrollo futuro de lo creado ${ }^{27}$. En ese mismo instante de producción divina, no sólo fueron hechos determinados seres sino todos los posibles seres que advendrán en el fututo, "Me preguntará. ¿De qué modo? Y responderé: invisible, potencialmente, en sus causas, como son creados los seres futuros aún no hechos" 28 .

El relato de seis días muestra de manera pedagógica el desarrollo que se da en el tiempo de los diferentes seres de la naturaleza. Las hierbas y árboles surgen de la tierra, como también los reptiles, asimismo las aves y peces desde agua e incluso el cuerpo del hombre fue formado desde lo ya existente. De esta manera se evidencia la duración que tiene el proceso de despliegue de las virtualidades contenidas en la creación. Por tanto, en el movimiento del mundo natural no hay nada que sea absolutamente nuevo, sino que los seres han sido creados en el principio por la acción divina, aunque deben desarrollarse y aparecer en el tiempo. Si no existen actualmente debemos entender que se encuentran latentes en los seres ahora existentes. Incluso ese mismo desarrollo ha sido establecido por el Creador, con su infinita inteligencia. Las rationes seminales son instrumentos por los cuales el Creador dispone el desarrollo y aparición de nuevas formalidades. Y así se entiende el orden que percibimos en el mundo natural, por ejemplo, cuando un animal engendra a otro de su misma especie. Ellas explican la generación de nuevas formas, pero también el orden establecido en la materia para su producción.

${ }^{26}$ Agustín de Hipona, Comentario literal al Génesis, op. cit., IV 33.51.

${ }^{27}$ Cfr. un resumen en T. Alesanco Reinares, Filosofía de San Agustín, Madrid, Augustinus, 2004, pp. 295-302.

${ }^{28}$ Agustín de Hipona, Comentario literal al Génesis, op. cit., VI 6.10. 
Si queremos entender esta doctrina como una referencia a la evolución, debemos ser cuidadosos ${ }^{29}$. Ya dijimos que, para Agustín, Dios ha creado todo lo que existe y establecido de manera latente las rationes seminales de los seres futuros. Por un lado, ellas explican la aparición de novedades en los seres y en su estructuración: "es de hecho evidente que, conforme a las especies que creó en el origen, hace ahora muchas nuevas que no hizo entonces" ${ }^{30}$. Como primera conclusión, es evidente que no podemos identificar esta postura con un fijismo creacionista, para el Doctor de Hipona en la realidad material hay verdadero movimiento y mutación entre los seres creados.

Sin embargo, no debemos entenderlo tampoco a la manera evolucionista, ya que aclara el Hiponense, "[Dios] impulsa con un poder oculto todo el universo, y en virtud de ese impulso todas las criaturas son puestas en movimiento" ${ }^{1}$. En la obra Sobre de la Trinidad, se aclara cuál es la relación del desarrollo impulsado por el Creador a través de las rationes seminales y los factores ambientales externos: "todos los seres están originaria y primordialmente contenidos en la urdimbre maravillosa de los elementos y les basta encontrar un ambiente propicio para manifestarse" ${ }^{32}$. Por tanto, no se puede aplicar al Hiponenese cualquier doctrina que quiera introducir cambios en los seres desde factores externos como causas principales de las mutaciones ${ }^{33}$.

En cuanto a la terminología utilizada, no se deben confundir los términos de semilla y razón seminal. Dice Agustín, "las semillas presentan una cierta semejanza con esto [las razones seminales], a causa de que aquellas cosas que han de ser futuras en los seres están ya incluidas en ellas" ${ }^{34}$. Las rationes seminales se comparan metafóricamente con la semilla, que podría ser perceptible a los sentidos, como por ejemplo, la de un árbol, en la que se encuentran las distintas partes que se desarrollarán posteriormente. Sin embargo, las rationes, sin dejar de ser algo físico denotan características de tipo formal en los seres, a modo de virtualidades que serán desarrolladas

${ }^{29}$ Cfr. T. Jáñez Barrio, "San Agustín frente a Darwin: Creacionismo evolutivo de las razones seminales”, Apuntes Filosóficos 35 (2009) 11-49.

${ }^{30}$ Agustín de Hipona, Comentario literal al Génesis, op. cit., V 20.41.

${ }^{31}$ Ibidem.

${ }^{32}$ Agustín de Hipona, Tratado sobre la Santísima Trinidad, Madrid, BAC, 1956, III 9.16.

${ }^{33} \mathrm{Y}$ aún de manera más explícita, cfr. ibídem, "[se] pueden aplicar al exterior causas accidentales, las que, aunque no se digan naturales, se utilizan siempre conforme a las leyes de la naturaleza, haciendo brotar de su seno las energías latentes y adquiriendo desarrollo oportuno".

${ }^{34}$ Agustín de Hipona, Comentario literal al Génesis, op. cit., VI 6.11. 
de manera sucesiva en el tiempo. Del mismo modo, es como Duns Escoto busca precisar las nociones de semen y ratio seminalis, según la intención de Agustín en los escritos antes citados. Este detalle aparece en el tratamiento que hace en la Lectura in Sententiarum, y en las Reportata parisiensia, pero no se encuentra la intención de clarificar el vocabulario en sus Quaestiones super Metaphysicorum.

El semen es definido como "una substancia generable y corruptible, diferente de quien genera y de lo engendrado, intermedia entre uno y otro" 35 , y también "es un medio necesario para la generación" 36 . La ratio seminalis "es una cualidad activa, que la semilla tiene por el que la generó" 37 , además agrega que es "una cualidad propia de la forma del semen" 38 .

El Doctor Sutil, tomando posición frente a las teorías revisadas anteriormente, define la ratio seminalis como una cualidad necesaria de la forma substancial de las semillas, que deben comprenderse como los intermediarios necesarios de los que se sirve el Creador para producir nuevas formas, de seres similares a los que se han engendrado. Si bien estas definiciones son generales, a continuación analizará su aplicación a los distintos seres corpóreos, ya que algunos se generan de manera mediata, y otros no. Por tanto, habrá que explicar de qué manera se pueden entender las rationes seminales en los diferentes modos de generación natural.

\section{b. El análisis de la generación en vivientes}

El primer caso, donde se busca evaluar la existencia o no de las rationes seminales, es respecto de los seres naturales que producen algo similar a ellos mismos de modo mediato, como sucede con la generación de las plantas y los animales ${ }^{39}$, es decir, de los orgánicos vivientes inferiores. Estos seres producen el semen, como un medio necesario para la generación de otro individuo de la misma especie, esto es la reproducción por propagación ${ }^{40}$. Afirma Escoto que "la forma de tales semillas producidas inmediatamente no parece ser la intención principal de la naturaleza, sino [que el fin es] generar algo desde la semilla: pues tal forma se da en el proceso natural

\footnotetext{
${ }^{35}$ I. Duns Scotus, Lectura II, op. cit., d. 18 q. 1-2 n. 21.

${ }^{36}$ I. Duns Scotus, Reportata II parisiensia, París, Vivès, 1894, vol. XXIII, d.18 q. 1 n. 10.

${ }^{37}$ I. Duns Scotus, Lectura II, op. cit., d. 18 q. 1-2 n.19.

${ }^{38}$ Ibídem.

${ }^{39}$ Cfr. I. Duns Scotus, Lectura II, op. cit., d. 18 q. 1-2 n. 17-39; Reportata II, op. cit., d. 18 q. 1 n. 9; QQ. Metaph., op. cit., VII q. 12 n. 32-40.

${ }^{40}$ Cfr. I. Duns Scotus, Reportata II, op. cit., d. 18 q. 1 n. 9.
} 
por una vía [que va] desde lo imperfecto hasta lo perfecto" ${ }^{41}$. El semen es intermediario entre dos substancias perfectas.

Del texto anterior podemos extraer algunas claves para el análisis posterior. Las semillas son substancias con formas determinadas, así se puede entender el orden que hay en la generación de nuevos seres, los cuales son semejantes -similia- a sus progenitores. De hecho, un animal no proviene de un hombre ni el trigo de un haba ${ }^{42}$. Este orden está relacionado con la actividad causal de quienes están involucrados en la consecución del nuevo ser como se verá más adelante. También se hace notar que la vía de generación natural va de lo imperfecto a lo perfecto, así como la resolución de los vivientes en los elementos es un proceso contrario. Ahora bien, parece que la vía natural contradice el principio filosófico por el cual lo perfecto no surge de lo imperfecto. Una vez más, tendremos que recurrir al análisis de las causas concurrentes para solucionar la contradicción, que es solo aparente.

La generación de estos seres sigue un proceso particular, que es natural, es decir, propio de la constitución orgánica establecida en ellos, y que los conduce a producir otro individuo mediante un camino que requiere ciertos pasos intermedios. El Doctor Sutil nos propone como ejemplo de un proceso natural de este tipo "la nutrición, donde la carne no se produce instantáneamente desde el alimento, pues primero se convierte en un humor, luego en sangre y finalmente en carne" ${ }^{43}$. Esta substancia intermedia del proceso natural es la semilla, dotada de una fuerza intrínseca con la capacidad de transmutar, y que se encuentra en continuo movimiento hasta la generación de un nuevo individuo de su especie. Esta fuerza intrínseca, según Escoto, es lo que el Hiponense llama razón seminal. Porque los seres naturales "se generan por especies determinadas (como la planta no se genera de la semilla del animal, ni al revés). Por esto, conviene establecer alguna cualidad activa educida en la semilla, determinada y consiguiente a una forma determinada en la semilla, que cambia hacia su forma propia" ${ }^{44}$.

Sin embargo, esta fuerza intrínseca no es, para el Doctor Sutil, contemporánea con la materia ${ }^{45}$. La ratio seminalis es una cierta cualidad de la forma substancial del semen, esto quiere decir, que no ha existido antes que ella y que se corromperá naturalmente antes que la forma substancial del semen. No puede una característica accidental ser más perfecta que la

\footnotetext{
${ }^{41}$ I. Duns Scotus, Lectura II, op. cit., d.18 q. 1-2 n. 18.

${ }^{42}$ Cfr. Agustín de Hipona, Comentario literal al Génesis, op. cit., IX 17.32.

${ }^{43}$ I. Duns Scotus, Lectura II, op. cit., d. 18 q. 1-2 n. 19.

${ }^{44}$ Ibídem, n. 23.

${ }^{45}$ Cfr. ibidem n. 26.
} 
forma total a la que pertenece, por ello la ratio seminalis, en cuanto cualidad adecuada a la forma del semen, desaparecerá antes que él mismo. Y aún más, es por esa cualidad que la semilla actúa, estando en continuo movimiento de transmutación hacia una forma similar. Aquí nos deja ver Escoto su modo particular de entender las rationes seminales: son una guía en el proceso natural, a modo de fuerza directriz ${ }^{46}$, por la cual el semen actúa en busca de una forma más perfecta.

Al barajarse la posibilidad de que la ratio seminalis sea parte de la forma a educir en la materia y de esta manera pueda generar una nueva forma, el Escocés se contrapone al axioma filosófico ${ }^{47}$ según el cual no puede darse algo desde lo imperfecto a lo perfecto. Un accidente no puede ser más perfecto que la forma substancial. Incluso el mismo semen no es más perfecto que la nueva forma a producir, ya que esta última es la forma principal buscada en el proceso natural de generación. Por ello, tanto el semen como la ratio seminalis son intermedios entre la forma perfecta del progenitor y el engendrado, formas perfectas. Un agente puede obrar, continúa Escoto, de modo unívoco, cuando produce una forma de su misma especie, o de manera equívoca cuando produce una forma de distinta especie. En este último caso, el agente equívoco solo puede producir un efecto que sea menos perfecto que él mismo ${ }^{48}$, por la propia virtud de su obrar, de lo contrario el Creador sería menos perfecto que toda su creación y esto es absurdo.

Por tanto, señala que la cualidad activa -esto es, la ratio seminalis- de la forma del semen no es generativa de la forma del nuevo individuo sino alterativa, ya que "o todo el semen no existe cuando se genera el compuesto; o si algo del semen permanece, esto que permanece no puede producir la forma substancial, porque es más imperfecto" ${ }^{49}$. En efecto, como el semen es un medio en el proceso natural, cabe preguntarse si es posible que él mismo actúe para generar una nueva forma substancial de la especie, no ya de su misma especie sino similar al que produjo la semilla. Este punto es analizado con un fino análisis del orden en la causalidad ${ }^{50}$. Cuando hay al menos dos causas que intervienen en la producción de un efecto tercero, el orden entre ellas puede ser:

${ }^{46}$ Cfr. E. Gilson, Juan Duns Escoto, Pamplona, Eunsa, 2007, p. 466.

${ }^{47}$ Cfr. el análisis específico en I. Duns Scotus, Opus Oxoniense, París, Vivès, 1894, vol. XVI, IV d. 12 q. 3 n. 13.

${ }^{48}$ Cfr. I. Duns Scotus, Lectura II, op. cit., d. 15 q. única n. 31.

${ }^{49}$ I. Duns Scotus, Lectura II, op. cit., d. 18 q. 1-2 n. 36

${ }^{50}$ Cfr. I. Duns Scotus, Lectura II, op. cit., d. 18 q. 1-2 n. 31-34; Reportata II, op. cit., d. 18 q. 1 n. 11; también de manera general se explica en I. Duns Scotus, Lectura in I Sententiarum, Vaticano, Typis Polyglottis Vaticanis, 1960, vol. XVI, d. 2 n. 45-48. 
i. esencial [causae essentialiter ordinatae $]=$ cuando dos causas se vinculan para producir un efecto en común, actuando ambas como causa total, de modo que deben estar las dos presentes para que se produzca ese efecto. Este será necesario cuando las dos se encuentren actuando simultáneamente.

ii. accidental [causae accidentaliter ordinatae] = en el que coinciden dos causas pero basta con que sólo una obre para poder producir el efecto, que puede existir independientemente de la existencia de su causa, como el abuelo y el padre respecto del hijo de éste último.

El Doctor Sutil nos advierte que en el caso de la generación de los vivientes, por ejemplo padre-hijo, se da una causa accidentalmente ordenada. El semen del padre tiene la capacidad de obrar y a través de él se produce el efecto, es decir, el hijo. Por lo tanto, "aquella causa que solamente da el ser y la virtud para obrar, y después lo abandona para que actúe según su ser, es sólo causa accidentalmente ordenada" ${ }^{51}$. El progenitor no es una causa esencialmente ordenada junto con el semen, según Escoto, porque el primero produce al segundo y éste último obra por su ratio seminalis para alterar la forma transeúnte del semen en pos de la nueva que se generará al corromperse éste, por ello el progenitor ya no actúa como causa esencialmente ordenada. Este orden que se da en la generación natural se debe entender como la sucesiva producción de una nueva forma gracias a la corrupción de otra ${ }^{52}$.

El análisis de la generación tal como ha sido formulado hasta ahora, y la aclaración respecto del orden causal, le sirve al Escocés para salvar el mundo natural de caer en creaciones sucesivas. Richard Cross resume que Escoto entiende por creación "la producción $p$ de algún elemento o elementos por un agente $a$, de tal manera que no haya ningún otro elemento más allá de $a$ necesario para la producción de $p "{ }^{33}$. Por lo tanto solo la causalidad en un orden esencial puede entenderse como creación. Este punto deja abierta la argumentación para defender la producción de una nueva forma sin la necesidad de crearla, aspecto que ocurre continuamente en la naturaleza, mediante causas accidentalmente ordenadas.

Con todo lo dicho acerca de la generación en los vivientes, queda aún por resolver un problema crucial: “¿por qué hay un alma vegetativa en las plantas y un alma sensitiva en el animal?” 54 . Esta pregunta presenta dificul-

\footnotetext{
${ }^{51}$ I. Duns Scotus, Lectura II, op. cit., d. 18 q. 1-2 n. 33.

${ }_{52}$ Cfr. sobre un análisis de punto R. Cross, The physics of Duns Scotus, op. cit., p. 110.

${ }^{53}$ Ibídem, p. 45.

${ }^{54}$ I. Duns Scotus, Lectura II, op. cit., d. 18 q. 1-2 n. 36.
} 
tades por varios motivos, a saber, la producción de esos seres no es inmediata, el semen es una forma media que no genera una forma substancial sino que dispone - por la ratio seminalis - la alteración previa. Además, el semen se corrompe cuando se genera el compuesto, e incluso si algo de él permanece no puede obrar por su virtud ni por la del progenitor porque sería alcanzar una forma más perfecta que la suya, que ya se ha afirmado sería absurdo.

El Doctor Sutil agrega que, por diversos motivos $^{55}$, tampoco puede darse la generación de las formas substanciales de los distintos vivientes a partir de los cuerpos celestes (por ser inanimados ${ }^{56}$ y por tanto menos perfectos que los vivientes), ni por las inteligencias (alegando al artículo 30 de la condena hecha por Esteban Tempier, en 1277, y porque ellas influirían a través del movimiento local de los cuerpos celestes). Luego, son producidas inmediatamente por Dios ${ }^{57}$. La virtud divina no solo crea inmediatamente las almas de los seres vivientes, sino que también es la garantía de sus diferencias específicas. Las especies tendrían, según Escoto, su fundamento último en el Creador que dispone según su voluntad divina las esencias de los seres. Incluso, siguiendo a Galeno y Averroes, sostendrá que el dator formarum es el responsable de la especie de cada forma ${ }^{58}$.

\section{c. Las razones seminales respecto de los elementos y mixtos}

Habiendo analizado lo que sucede en los seres vivos, a continuación el Doctor Sutil estudia el ámbito de la materia inerte. Los hechos de experiencia que propone como ejemplos a estudiar pueden resumirse asís: el cuerpo celeste que educe una forma desde el cuerpo en putrefacción; los minerales que unidos forman una piedra; y la corrupción de los elementos entre sí (como el fuego que actúa sobre el agua para evaporarla). Estos

${ }^{55}$ Cfr. ibidem n. 38.

${ }^{56}$ Cfr. I. Duns Scotus, Lectura II, op. cit., d. 14 q. 4 n. $45-52$.

${ }^{57}$ Cfr. I. Duns Scotus, Lectura II, op. cit., d. 18 q. 1-2 n. 37-38. "Sed dices: a quo igitur est anima vegetativa in planta et sensitiva in animali? Dico quod ista quaestio non habet difficultatem quando forma est materialis et inanimate (...) Sed quando forma inducta est vitalis, tunc dubium est: non enim potest esse a generante nec a virtute in semine, propter ea quae dicta sunt; non a corpore caelesti, ponendo quod sit inanimatum, quia 'omne vivum perfectius est non vivo' secundum Augustinum, et numquam imperfectius producit perfectius; non ab intelligentia, propter illum articulum condemnatum; igitur a Deo". También Reportata II, op. cit., d. 18 q. 1 n. 11.

${ }^{58}$ Cfr. I. Duns Scotus, QQ. Meth. VII, op. cit., q. 12 n. 40, texto interpolado c.

${ }^{59} \mathrm{Cfr}$. ibídem n. 41. Evidentemente, los ejemplos se adecúan a los conocimientos propios del período histórico en el cuál se formó Duns Escoto. 
casos se podrían explicar por ciertas fuerzas que actúan para la realización de las transmutaciones citadas, y por lo tanto, serían comparables con la actividad desarrollada por las rationes seminales, según se ha explicado en el apartado anterior.

En las Quaestiones super Metaphysicorum, declara de manera definitiva que no hay ni se pueden entender las rationes seminales respecto de los cuerpos inanimados. Esto es así ya que, para obrar, estos necesitan de la presencia de un agente extrínseco, es decir, que sin el sol, del cuerpo putrefacto no puede darse un mixto, y sin el fuego el agua volverá al estado que le es propio. Sólo pueden entenderse las cualidades activas alterativas - rationes seminales - en los casos donde la producción de una nueva forma conlleva un proceso y cuando el agente extrínseco encontrándose ausente, ese proceso continúa su curso ${ }^{60}$ guiado por aquellas rationes.

Sin embargo, en la Lectura y en las Reportata, el Doctor Sutil se muestra más conciliador. Habiendo establecido en el caso de los vivientes que las razones seminales son formas medias, y dado que también existen cambios entre los inanimados, conviene reconocer, paralelamente, cierta semejanza con el proceso mediato de generación de los seres vivos. Sin embargo, esta comparación presenta dificultades, ya que en el caso de los cuerpos celestes, ellos educen una forma de distinta especie a la propia y en el caso de los elementos entre sí no hay formas medias, sino que la producción se realiza de manera inmediata. Pero, ¿qué podemos decir de los elementos respecto de los cuerpos mixtos? Desde aquí, Duns Escoto, retoma la sentencia que expresa Agustín en Sobre la Trinidad, "en los elementos de este mundo están latentes las razones seminales respecto de todas las cosas que serán hechas a partir de ellos" ${ }^{61}$. En el caso de los mixtos imperfectos, o mezclas, como las nieves y lluvias, donde el elemento conserva su naturaleza, durante el proceso de formación del mixto podemos llamar semen al mixto medio y razón seminal a su cualidad activa. También sucede esto de modo análogo en la formación de los mixtos perfectos, es decir, las combinaciones.

El proceso natural en los seres inertes se puede explicar de la siguiente manera, "cuando el mixto deber ser generado desde un elemento (tal el caso de una piedra o un compuesto que se pueda corromper), primero se educe alguna cualidad proporcionada a ese mixto, y finalmente la forma del elemento se corrompe" ${ }^{62}$, el cambio se inicia en un orden accidental y luego alcanza la forma. El cuerpo celeste, por ser más noble, puede educir

${ }^{60}$ Cfr. ibídem n. 44.

${ }^{61}$ Agustín de Hipona, Sobre la Trinidad, op. cit., III 8.13.

${ }^{62}$ I. Duns Scotus, Lectura II, op. cit., d.18 q. 1-2 n.43. 
a la cualidad activa que posibilita alterar la forma del elemento y educir la forma substancial del mixto que se generará6 ${ }^{63}$. De esta manera, se puede entender que el elemento funcione a modo de semen y la cualidad activa alterativa que el cuerpo celeste educe, obre a modo de ratio seminalis ${ }^{64}$. Un punto interesante para aclarar de este caso es que aquella acción la puede realizar el cuerpo noble, como lo aclara Escoto, gracias a la concurrencia de la actividad del agens universalis, esto es, Dios ${ }^{65}$. También podemos decir —asegura Escoto- que si son muchos los elementos que concurren para la formación de un compuesto, serán muchas las semillas que lo producen: “y así, [cada] elemento - en cuanto término `a quó - es semen respecto del mixto, y la fuerza activa en [cada] elemento es razón seminal" ${ }^{66}$. Los compuestos materiales — según la doctrina escotista- que se forman a partir de los elementos, son una nueva realidad física unitaria. Los elementos no permanecen según sus esencias completas ni como cualidades remisas, sino virtualmente, dando así ciertas cualidades a los mixtos y posibilitando la futura resolución en ellos ${ }^{67}$. En este caso tampoco se puede establecer una causalidad de orden esencial entre los elementos respecto del mixto, por ello el Doctor Sutil vuelve a introducir como causa principal concurrente al agente universal, quien es capaz de educir las cualidades activas en los elementos hacia la corrupción de ellos y generación del mixto ${ }^{68}$.

Queda por resolver una cuestión más. Teniendo en cuenta la doctrina aristotélica sobre la generación recíproca de los elementos ${ }^{69}$, Escoto se pregunta si "¿acaso es necesario establecer las razones seminales [en el orden del cambio] respecto de los elementos [entre sí]?"70. La respuesta es negativa ya que la sentencia de Agustín se refiere a las cosas que serán hechas a partir de los elementos, esto es, a los mixtos. Con todo, el filósofo escocés da varios argumentos para mostrar la inconveniencia de la propuesta. Habiendo establecido que la ratio seminalis actúa como guía, se debe recordar que obra cuando el agente que produce la semilla está ausente, y así se garantiza el proceso natural. Ahora bien, en la generación de los elementos — por ejemplo- cuando el agua se calienta por acción del fuego (perdiendo

${ }^{63}$ Sin embargo, Duns Escoto niega la posibilidad de que el sol genere el alma de los vivientes. Cfr. G. Sondag, "Duns Scot sur les raisons séminales”, Archa Verbi 5 (2010) 201.

${ }^{64}$ Cfr. I. Duns Scotus, Lectura II, op. cit., d. 18 q. 1-2 n. 47.

${ }^{65}$ Cfr. ibídem n. 46.

${ }^{66}$ Ibidem n. 49.

${ }^{67}$ Cfr. I. Duns Scotus, Lectura II, op. cit., d. 15 q. única n. 26-35.

${ }^{68}$ Cfr. I. Duns Scotus, Lectura II, op. cit., d. 18 q. 1-2 n. 50; d. 15 q. única n. 35.

${ }^{69}$ Cfr. Aristóteles, Acerca de la generación y la corrupción, Madrid, Gredos, 1998, II 4.

${ }^{70}$ I. Duns Scotus, Lectura II, op. cit., d. 18 q. 1-2 n. 5. 
la cualidad propia frío y ganando la cualidad caliente), si éste falta no continúa el agua el proceso hacia la forma del fuego, sino que se vuelve hacia su cualidad natural, y se enfría. Esto da cuenta de la no existencia de una forma intermedia que posea la potencialidad de alterar por medio de una cualidad activa al agua, y esto se puede aplicar a los demás elementos. En conclusión, no hay tales razones seminales en la mutua generación de ellos, es decir, que se da de manera inmediata.

Además, como se dijo antes, la forma del semen es más imperfecta que la forma del compuesto a generarse, porque en el proceso natural hay cierta direccionalidad hacia esta forma que se consigue gracias a las semillas como intermediarias. Pero si se analiza el caso de los elementos, ellos son formas especificas infimas ${ }^{71}$, y si se transmutan en otro elemento, éste también será una forma de tal característica. Por tanto, siendo el semen una forma no buscada principalmente por la naturaleza, no se podrá comparar al elemento ni con él ni con la ratio seminalis, puesto que demandarían ser formas más imperfectas y por lo tanto inferiores. También se argumenta que "el semen no se da sino en aquellos seres que generan mediatamente [algo] similar a ellos, pero en cambio un elemento inmediatamente genera a otro [distinto de sí]"72. Por lo que no se puede asignar semen ni ratio seminalis en el caso de cambio entre los elementos. Sin embargo, el Doctor Sutil advierte que per extensionem del término razón seminal se puede entender cualquier virtud activa, y de esta manera el fuego respecto del agua puede decirse razón seminal, aclarando que este uso amplio no se ajusta a la intención del Hiponense.

Para finalizar, podemos concluir en primer lugar que hay un cierto abandono del pensamiento agustiniano sobre la doctrina de las rationes seminales, o mejor, una reinterpretación para ajustarla al modo en que la propia filosofía natural de Escoto es desarrollada. La preeminencia del aspecto formal en todo su pensamiento y la materia entendida como una cierta realidad actual, le conducen a reducir la doctrina revisada en el presente artículo a una cuestión secundaria. En efecto, en el único caso en que se hace necesario afirmar - de manera estricta - la existencia de semen y ratio seminalis es en le caso de los vivientes, ya que pueden reproducir una nueva

\footnotetext{
${ }^{71}$ Cfr. ibidem n. 53.

${ }^{72}$ Ibidem n. 54.
} 
forma de su misma especie. De esta manera, la actividad productiva de los seres vivos es guiada en su materialidad por las razones seminales.

Sin embargo, el Doctor Sutil no puede justificar de manera natural la producción del alma en los vivientes imperfectos, y por este motivo recurre, a nuestro juicio, a la necesidad de afirmar que las almas proceden directamente de Dios. El principio de parsimonia, que impide introducir explicaciones innecesarias, casi se ve quebrado por la acción creadora en el caso de esos seres. Esto podría ser justificado por una impronta voluntarista en la actividad del Creador, que domina la exposición de Escoto, y donde se manifiesta que un principio teológico asume el control de la especulación. Por lo demás, esta doctrina le permitirá explicar en sede antropológica la creación del alma racional en un cuerpo humano. 